and in $G$ must separate $G$. But by Theorem I this set is 0-dimensional, a contradiction. Hence some element of $G$ intersects each of $D_{1}$ and $D_{2}$, and thus some element of $G^{\prime}$ contains an arc $h$ with endpoints in $D_{1}$ and $D_{2}$ respectively. But by an argument similar to that used in the proof of Theorem I we can exhibit an open subcollection $U$ of $G$ with $U$ containing $g$ and with $\bar{U}-U$ countable, a contradiction.

\title{
REFERENCES
}

1. R. D. Anderson, Continuous collections of continuous curves, Duke Math. J. vol. 21 (1954) pp. 363-368.

2. Witold Hurewicz and Henry Wallman, Dimension theory, Princeton University Press, 1948.

3. J. H. Roberts, Collections filling a plane, Duke Math. J. vol. 2 (1936) pp. 10-19.

The University of Pennsylvania and

Goucher College

\section{A NOTE ON BASIC SETS OF HOMOGENEOUS HARMONIC POLYNOMIALS}

E. P. MILES, JR. AND E. WILliamS

For any set of non-negative integers $\left(b_{j}\right)$ such that $b_{1} \leqq 1$ and $\sum_{j=1}^{k} b_{j}=n$, let

$$
H_{b_{1} \ldots b_{k}}^{n}=\sum(-1)^{\left[a_{1} / 2\right]} \frac{n !}{\prod_{j=1}^{k} a_{j} !} \cdot \frac{\left[\frac{a_{1}}{2}\right] !}{\prod_{j=2}^{k}\left(\frac{b_{j}-a_{j}}{2}\right) !} \prod_{j=1}^{k} x_{j}^{a_{j}}
$$

where the summation is extended over all $\left(a_{j}\right)$ such that,

(a) $a_{j} \equiv b_{j} \bmod 2, j=1,2, \cdots, k$,

(b) $\sum_{j=1}^{\boldsymbol{k}} a_{j}=n$,

(c) $a_{j} \leqq b_{j}, j=2,3, \cdots, k$.

The polynomials (1) were shown by the authors to form a basic set of homogeneous harmonic polynomials in $k$ variables [1]. ${ }^{1}$

It is easily seen that the following differential recursion formulas hold for these polynomials:

Presented to the Society, November 26, 1954 under the title $A$ basic set of homogeneous polynomials in $k$ variables. II; received by the editors November 29, 1954.

${ }^{1}$ Numbers in brackets refer to bibliography at the end of the paper. 
(2)

$$
\frac{\partial}{\partial x_{1}} H_{1 b_{2} \cdots b_{k}}^{n}=n H_{0 b_{2} \cdots b_{k}}^{n-1}, \quad 1+\sum_{j=2}^{k} b_{j}=n ;
$$

$\frac{\partial}{\partial x_{j}} H_{b_{1} b_{2} \cdots b_{j} \cdots b_{k}}^{n}=n H_{b_{1} b_{2} \cdots\left(b_{j}-1\right) \cdots b_{k}}^{n-1}$,

$$
j=2,3, \cdots, k, b_{1}=0,1, \sum_{j=1}^{k} b_{j}=n ;
$$

(4) $\frac{\partial}{\partial x_{1}} H_{0 b_{2} \cdots b_{j} \cdots b_{k}}^{n}=-n \sum_{j=2}^{k} H_{1 b_{2} \cdots\left(b_{j}-2\right) \cdots b_{k}}^{n-1}$, $\sum_{j=2}^{k} b_{j}=n$

where $H_{c_{1} c_{2}}^{n} \cdots c_{j} \cdots c_{k}=0$ if $c_{j}<0$.

The relations (2) and ( 3 ) follow directly from (1) by differentiation. To prove (4) we note from (2) that

$$
\frac{\partial^{2}}{\partial x_{1}^{2}} H_{1 b_{2} \cdots b_{k}}^{n+1}=(n+1) \frac{\partial}{\partial x_{1}} H_{0 b_{2} \cdots b_{k}}^{n}
$$

and from (3) that

$$
\frac{\partial^{2}}{\partial x_{j}^{2}} H_{1 b_{2} \cdots b_{j} \cdots b_{k}}^{n+1}=(n+1) n H_{1 b_{2} \cdots\left(b_{j}-2\right) \cdots b_{k}}^{n-1}, \quad j=2, \cdots, k .
$$

Combining (5) and (6) and noting that $H_{1 b_{2} \cdots b_{k}}^{n+1}$ is harmonic, we obtain (4).

For $k=2$ the basic sets (1) give the real and imaginary parts of $\left(x_{1}+i x_{2}\right)^{n}$. For $k=3$ they give a single formulation for the eight types first given by Protter [2] and later reduced by him to four types [3]. The differential recursion formulas generalize those given by Protter. The authors are indebted to Rosenbloom and Bers for calling their attention to these results of Protter.

\section{BIBLIOGRAPHY}

1. E. P. Miles, Jr. and Ernest Williams, $A$ basic set of homogeneous harmonic polynomials in $k$ variables, Proc. Amer. Math. Soc. vol. 6 (1955) pp. 191-194.

2. M. H. Protter, Generalized spherical harmonics, Trans. Amer. Math. Soc. vol. 63 (1948) pp. 314-341.

3. - On a class of harmonic polynomials, Portugaliae Mathematica vol. 10 (1951) pp. 11-22.

Alabama Polytechnic Institute 